\title{
Discovery of Novel Pseudomonas putida Flavin-Binding Fluorescent Protein Variants with Significantly Improved Quantum Yield
}

\author{
Sanghwan Ko ${ }^{1,2}$, Hyunwoo Jeon ${ }^{3}$, Sanghan Yoon ${ }^{3}$, Munsu Kyung ${ }^{1}$, Hyungdon Yun ${ }^{3}$, \\ Jung-Hyun $\mathrm{Na}^{4,}{ }^{,}$, Sang Taek Jung ${ }^{1, *}$
}

${ }^{1}$ Department of Biomedical Sciences, Graduate School of Medicine, Korea University, Seoul 02841, Korea

${ }^{2}$ Department of Applied Chemistry, Kookmin University, Seoul 02707, Korea

${ }^{3}$ Department of Bioscience and Biotechnology, Konkuk University, Seoul 05029, Korea

${ }^{4}$ Department of Pharmaceutical Engineering, Sangji University, Wonju 26339, Korea

*Correspondence to:

Sang Taek Jung (E-mail: sjung@korea.ac.kr)

Jung-Hyun Na (E-mail: najunghyun@sangji.ac.kr)

Contract grant sponsor: Ministry of Science and ICT

Contract grant numbers: 2019R1F1A1059834, 2019R1A4A1029000, and 2017M3A9C8060552 
Supporting Information Table 1. FMN binding cavity volume of SB2 variants.

\begin{tabular}{cc}
\hline SB variants & $\begin{array}{c}\text { Cavity volume } \\
\left(\AA^{3}\right)\end{array}$ \\
\hline KOFP-13 & 764 \\
KOFP-13-C53G & 863 \\
\hline
\end{tabular}

${ }^{*}$ Cavity volume of SB variants was calculated by CB-dock server. ${ }^{1}$ 


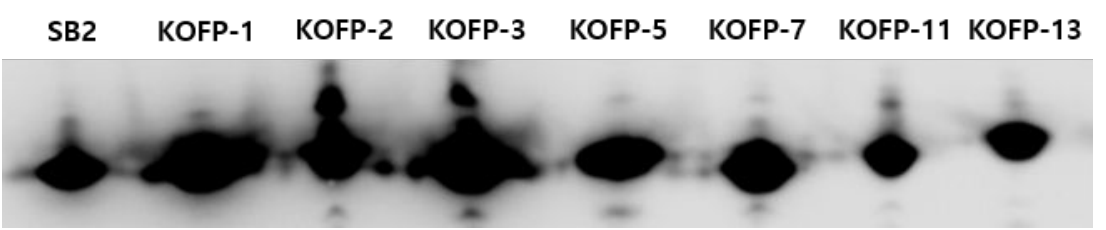

Supporting Information Figure 1. Western blot analysis showing the expression level of isolated SB2 variants compared to that of SB2. 
A

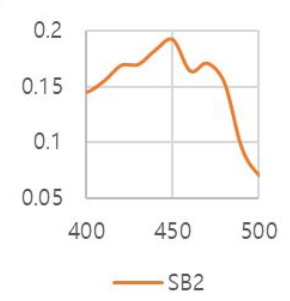

E

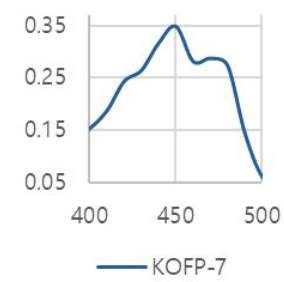

I
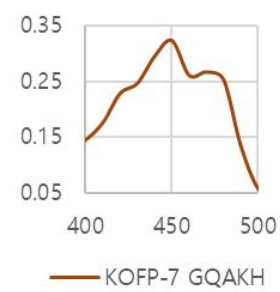

B
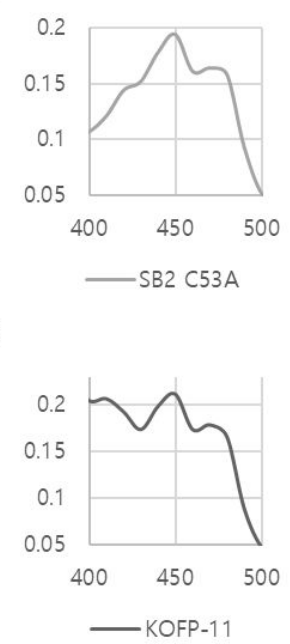

J

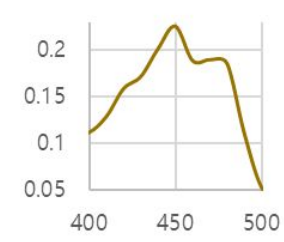

- KOFP-11 C53G
C

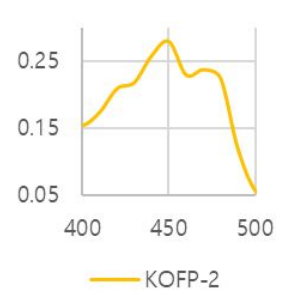

G

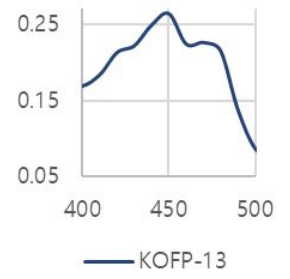

K

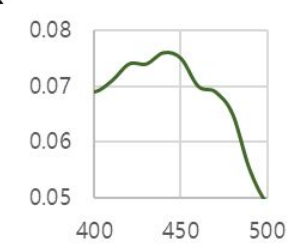

-KOFP-13 C53G
D

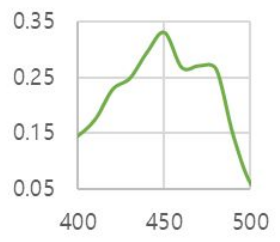

$\mathrm{H}$

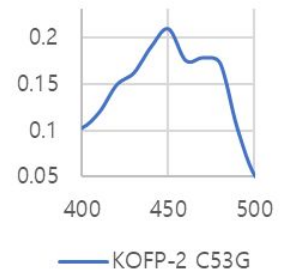

L

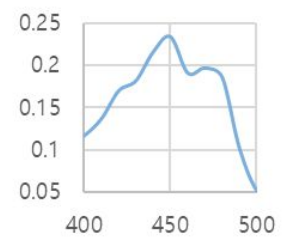

—SB2 Q23P/C53G/Y112F
M

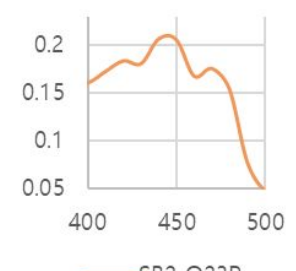

Q

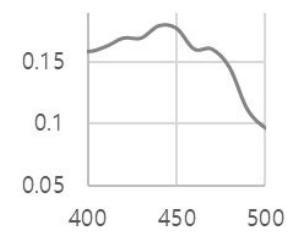

—SB2 Q108R

U

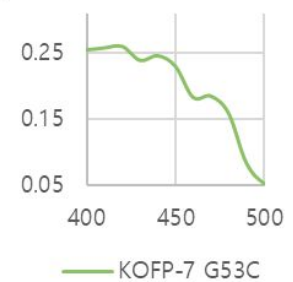

N

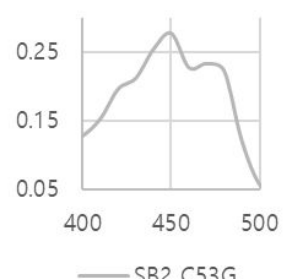

R

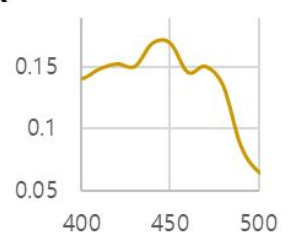

-SB2 I115T

V

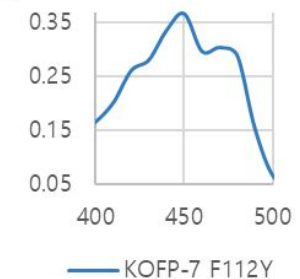

0

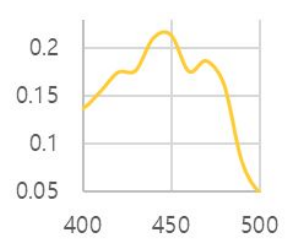

S

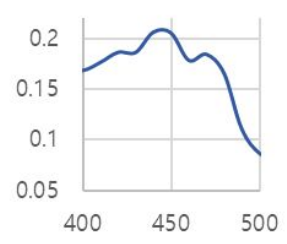

-SB2 V129M
P

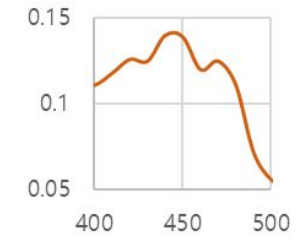

T

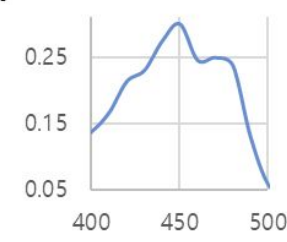


Supporting Information Figure 2. Absorption spectra of isolated SB2 variants. (A) SB2, (B) SB2 C53A, (C) KOFP-2, (D) KOFP-5, (E) KOFP-7, (F) KOFP-11, (G) KOFP-13, (H) KOFP-2 C53G, (I) KOFP-7 GQAKH, (J) KOFP-11 C53G, (K) KOFP-13 C53G, (L) SB2 Q23P/C53G/Y112F, (M) SB2 Q23P, (N) SB2 C53G, (O) SB2 Y112F, (P) SB2 H13Y, (Q) SB2 Q108R, (R) SB2 I115T, (S) SB2 V129M, (T) KOFP-7 P23Q, (U) KOFP-7 G53C, (V) KOFP-7 F112Y. 


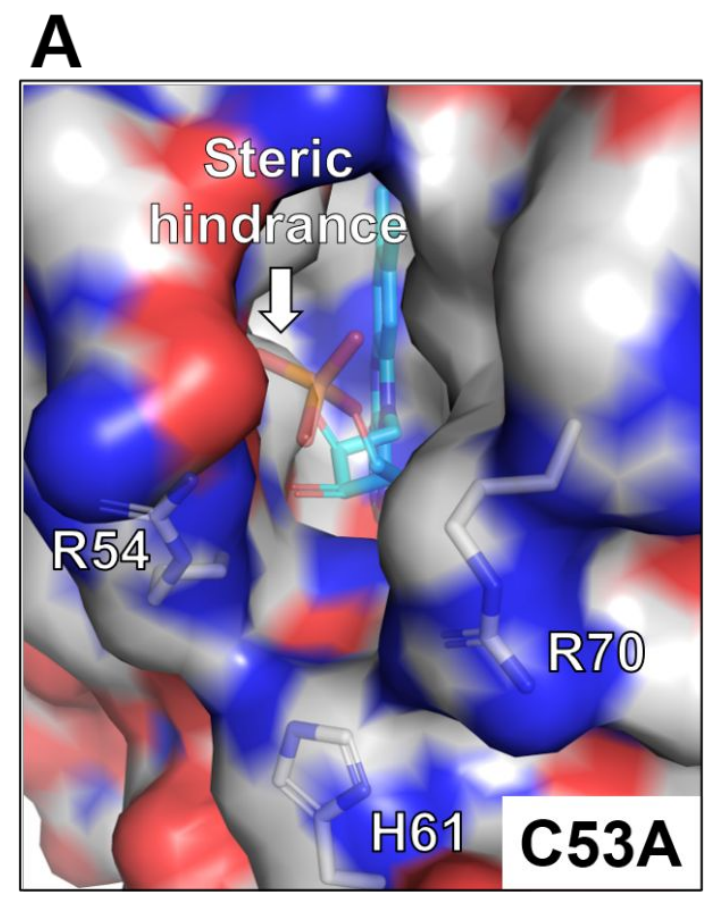

\section{B}

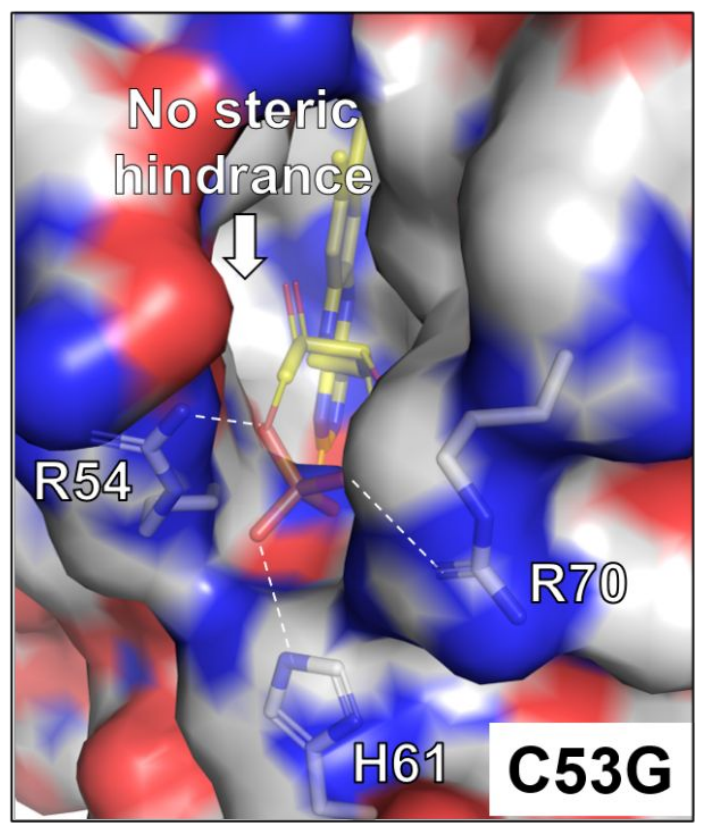

Supporting Information Figure 3. Electron density maps of in silico SB2 C53A/ (A) and C53G/FMN (B) complex models. The residues of SB2 variants (gray), FMNs of SB2 C53A (cyan), and C53G (yellow) are represented by sticks. Nitrogen, oxygen, and phosphorus atoms are colored blue, red, and orange, respectively. White arrow indicates the 53rd amino acid position, and dotted white lines indicate interatomic interactions. 


\section{References}

1. Liu, Y.; Grimm, M.; Dai, W. T.; Hou, M. C.; Xiao, Z. X.; Cao, Y., CB-Dock: a web server for cavity detection-guided protein-ligand blind docking. Acta Pharmacol. Sin. 2020, 41 (1), 138-144. 